Check for updates

Cite this: RSC Chem. Biol., 2021, 2, 248

Received 17th August 2020 Accepted 12th November 2020

DOI: 10.1039/d0cb00152j

rsc.li/rsc-chembio

\section{Fluorescence lifetime predicts performance of voltage sensitive fluorophores in cardiomyocytes and neurons $\dagger$}

\author{
Steven C. Boggess, (D) $\ddagger^{a}$ Julia R. Lazzari-Dean, (D) $\ddagger^{a}$ Benjamin K. Raliski, $\mathbb{D} \ddagger^{a}$ \\ Dong Min Mun, ${ }^{a}$ Amy Y. Li, ${ }^{a}$ Joshua L. Turnbull ${ }^{a}$ and Evan W. Miller (D) *abc
}

\begin{abstract}
Voltage imaging with fluorescent indicators offers a powerful complement to traditional electrode or $\mathrm{Ca}^{2+}$ imaging approaches for monitoring electrical activity. Small molecule fluorescent indicators present the unique opportunity for exquisite control over molecular structure, enabling detailed investigations of structure/function relationships. In this paper, we tune the conjugation between aniline donors and aromatic $\pi$ systems within the context of photoinduced electron transfer $(\mathrm{PeT})$ based voltage indicators. We describe the design and synthesis of four new voltage-sensitive fluorophores (VoltageFluors, or VFs). Three of these dyes have higher relative voltage sensitivities $(\Delta F / F)$ than the previously-reported indicator, VF2.1.Cl. We pair these new indicators with existing VFs to construct a library of voltage indicators with varying degrees of conjugation between the aniline nitrogen lone pair and the aromatic $\pi$ system. Using a combination of steady-state and time-resolved fluorescence spectroscopy, cellular electrophysiology, fluorescence lifetime imaging microscopy (FLIM), and functional imaging in mammalian neurons and human cardiomyocytes, we establish a detailed link between the photophysical properties of VF dyes and their ability to report on membrane potential dynamics with high signal-to-noise. Anilines with intermediate degrees of conjugation to the aromatic $\pi$ system experience intermediate rates of PeT and possess the highest absolute voltage sensitivities. Measured using FLIM in patch-clamped HEK cells, we find that the absolute voltage sensitivity of fluorescence lifetime $\left(\Delta \tau_{\mathrm{fl}}\right.$ per $\left.\mathrm{mV}\right)$, coupled with traditional fluorescence intensity-based metrics like $\Delta F / F$ and signal-to-noise ratio (SNR), provides a powerful method to both predict and understand indicator performance in cellular systems.
\end{abstract}

\section{Introduction}

Cell membrane potential $\left(V_{\text {mem }}\right)$ arises from an unequal distribution of ions across a selectively permeable lipid bilayer. In excitable cells such as neurons and cardiomyocytes, $V_{\text {mem }}$ fluctuates on the order of milliseconds to create action potentials (APs). These APs facilitate electrochemical communication across synapses and coordinate the contraction of millions of cells across the chambers of the heart. Measuring this electrical activity is critical to understanding cell physiology in health and disease. The gold standard for measuring $V_{\text {mem }}$ is

\footnotetext{
${ }^{a}$ Department of Chemistry, University of California, Berkeley, California 94720 , USA

${ }^{b}$ Department of Molecular \& Cell Biology, University of California, Berkeley, California 94720, USA. E-mail: evanwmiller@berkeley.edu

${ }^{c}$ Helen Wills Neuroscience Institute, University of California, Berkeley, California 94720, USA

$\dagger$ Electronic supplementary information (ESI) available: Including supporting figures, spectra, procedures, and analysis. See DOI: 10.1039/d0cb00152j

\$ These authors contributed equally and are listed in alphabetical order.
}

patch-clamp electrophysiology, a series of techniques that use an electrode in direct contact with the cell of interest, allowing very precise measurement of $V_{\text {mem }}$. However, the lowthroughput, high invasiveness, and low spatial resolution ${ }^{1}$ of patch-clamp electrophysiology render it an incomplete $V_{\text {mem }}$ measurement technique.

To record electrical activity in a highly multiplexed and less invasive manner, our lab and others have undertaken the development of fluorescent voltage indicators, ${ }^{2-4}$ either as genetically encoded voltage indicators or small molecule voltage sensitive dyes. Fluorescent voltage indicators for action potential detection should possess fast response kinetics to respond to rapid ( $<1 \mathrm{~ms}$ ) $V_{\text {mem }}$ changes, bright fluorophores, good membrane localization, and high sensitivity to $V_{\text {mem }}$ changes. ${ }^{5,6}$ Design of indicators with all of these characteristics remains a substantial challenge.

The precise molecular control over small molecules provided by synthetic chemistry is an advantage compared to the design or evolution of fluorescent proteins. Control of the structure of fluorophores has facilitated major advances 
a)

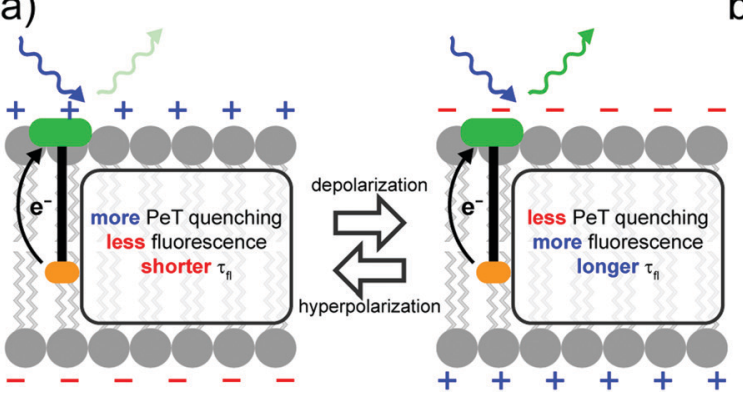

b)

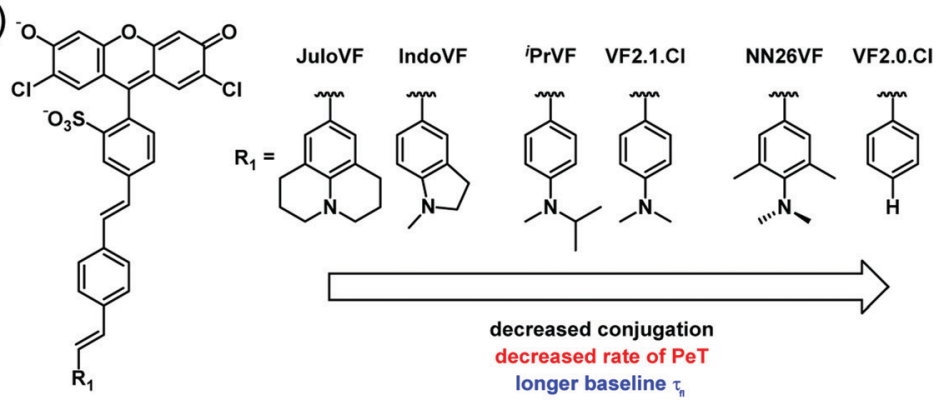

Scheme 1 Proposed mechanism for VoltageFluor indicators and modified anline library.

in biological sensing: small molecule indicators of $\mathrm{Ca}^{2+}$ revolutionized physiology, ${ }^{7,8}$ simple modifications of the fluorophore structure profoundly alter color $^{9-15}$ or improve brightness, ${ }^{16,17}$ and innovative functionality is opening new vistas in superresolution and long-term microscopy. ${ }^{18-20}$ Optimization of the brightness, sensitivity, color, and localization of small molecule voltage-sensitive indicators dyes has been fruitful, ${ }^{21-24}$ but further investigation is required to obtain probes with sufficient signalto-noise ratios for single-cell recordings in thick tissue.

Our lab has developed small molecule voltage sensitive dyes (VoltageFluors, VFs) based on a photoinduced electron transfer (PeT)-based $V_{\text {mem }}$ sensing through a conjugated molecular wire (Scheme 1a). Because PeT occurs from a membrane-localized aniline electron donor through a conjugated $\pi$ system, it is sensitive to the transmembrane electric field (Scheme 1a). We previously demonstrated that the absorption and emission wavelength of VFs can be tuned through the incorporation of different chromophores, ${ }^{24-27}$ and the voltage sensitivity can be modulated via optimization of the orientation ${ }^{28}$ oxidation potential, ${ }^{23}$ and identity of the molecular wire. ${ }^{29}$

In this work, we investigated the extent of conjugation between the aniline donor and the molecular wire as a strategy for tuning VFs. Using VF2.1.Cl ${ }^{30}$ and VF2.0. $\mathrm{Cl}^{23}$ as reference structures, we designed and synthesized four indicators with varying degrees of conjugation between the aniline donor and the molecular wire (Scheme 1b). We designed JuloVF (17) and IndoVF (18) to contain annulated anilines with higher degrees of conjugation than the dimethyl aniline in VF2.1.Cl (Scheme 1b). In contrast, we designed NN26VF (20) to contain an aniline locked out-of-plane by steric repulsion that we could compare to the compound VF2.0.Cl, which completely lacks an aniline group (Scheme 1b). Finally, we designed ${ }^{\mathrm{i}} \operatorname{PrVF}(\mathbf{1 9})$ to be similar to VF2.1.Cl but with a bulkier aniline group to investigate how increased substitution on the aniline would affect conjugation to the molecular wire (Scheme $1 \mathrm{~b}$ ). We hypothesized that as aniline conjugation decreased in the VF dye series, the rate of PeT would also decrease (Scheme 1b).

We previously showed that membrane potential-induced changes in the rate of PeT in VF2.1.Cl also produce changes in the fluorescence lifetime $\left(\tau_{\mathrm{fl}}\right)$, which can be used to read out absolute $V_{\mathrm{mem}}{ }^{31}$ This allowed us to make an optical estimation of the $\mathrm{mV}$ value of $V_{\mathrm{mem}}$, without the confounds associated with intensity-based or ratiometric imaging. ${ }^{31}$ In the context of probe design, measuring fluorescence lifetime changes allows measurement of the absolute voltage sensitivity $\left(\Delta \tau_{\mathrm{fl}}\right.$ per $\left.\mathrm{mV}\right)$ for a dye species, as changes in lifetime do not depend on the probe concentration. ${ }^{32}$ By measuring the baseline $\tau_{\mathrm{fl}}$, and the $V_{\text {mem }}$ dependent $\tau_{\mathrm{fl}}$ change, we can determine differences in voltage-sensitive PeT between VF dyes in living cells, rather than in a cuvette. Because of their capacity to provide insights on fundamental photophysical parameters, like fluorescence quantum yield, $\tau_{\mathrm{fl}}$ measurements are an important complement alongside intensity-based methods for benchmarking indicator behavior, such as relative voltage sensitivity $(\% \Delta F / F)$ and signal-to-noise ratio (SNR).

With this library of six aniline-modified VFs, we investigated the response of fluorescence intensity and fluorescence lifetime to changes in $V_{\mathrm{mem}}$. We find that modifying aniline conjugation in $\mathrm{VF}$ dyes tunes relative voltage sensitivity $(\% \Delta F / F)$, signal-tonoise ratio (SNR), absolute voltage sensitivity $\left(\Delta \tau_{\mathrm{fl}}\right.$ per $\left.\mathrm{mV}\right)$, and baseline lifetime $\left(\tau_{\mathrm{fl}}\right)$ across an order of magnitude. We then compared the ability of three VF dyes to measure cardiac and neuronal action potentials.

VF dyes displaying the largest relative sensitivities $(\% \Delta F / F)$ do not have the best performance in action potential detection; rather, indicators that have the highest SNR in HEK cells perform best in excitable cells. Along with SNR, the absolute voltage sensitivity of the fluorescence lifetime $\left(\Delta \tau_{\mathrm{fl}}\right.$ per $\left.\mathrm{mV}\right)$ ably predicts the performance of VF dyes in excitable cells. Alongside fluorescence intensity-based metrics, determination of $\Delta \tau_{\mathrm{fl}}$ per $\mathrm{mV}$ provides a means for both evaluating and understating indicator performance in detection of physiologically relevant $V_{\text {mem }}$ signals. We propose that absolute voltage sensitivity of the fluorescence lifetime can be incorporated as a standard for evaluating future indicator derivatives.

\section{Results}

To examine the relationships between aniline modification and voltage sensitivity, we synthesized phenylene-vinylene molecular wires with julolidine, $N$-methylindoline, $N$-methyl- $N$-isopropyl, and $N, N, 2,6$-tetramethylaniline aniline donors (Scheme 2). Starting with julolidine, Vilsmeier-Haack formylation with $\mathrm{POCl}_{3}$ was used to obtain benzaldehyde 1 in $88 \%$ yield (Scheme S1, ESI $\dagger$ ). This was converted to the aminostyrene by Wittig olefination and was promptly reacted with 4-bromobenzaldhyde in a palladium 


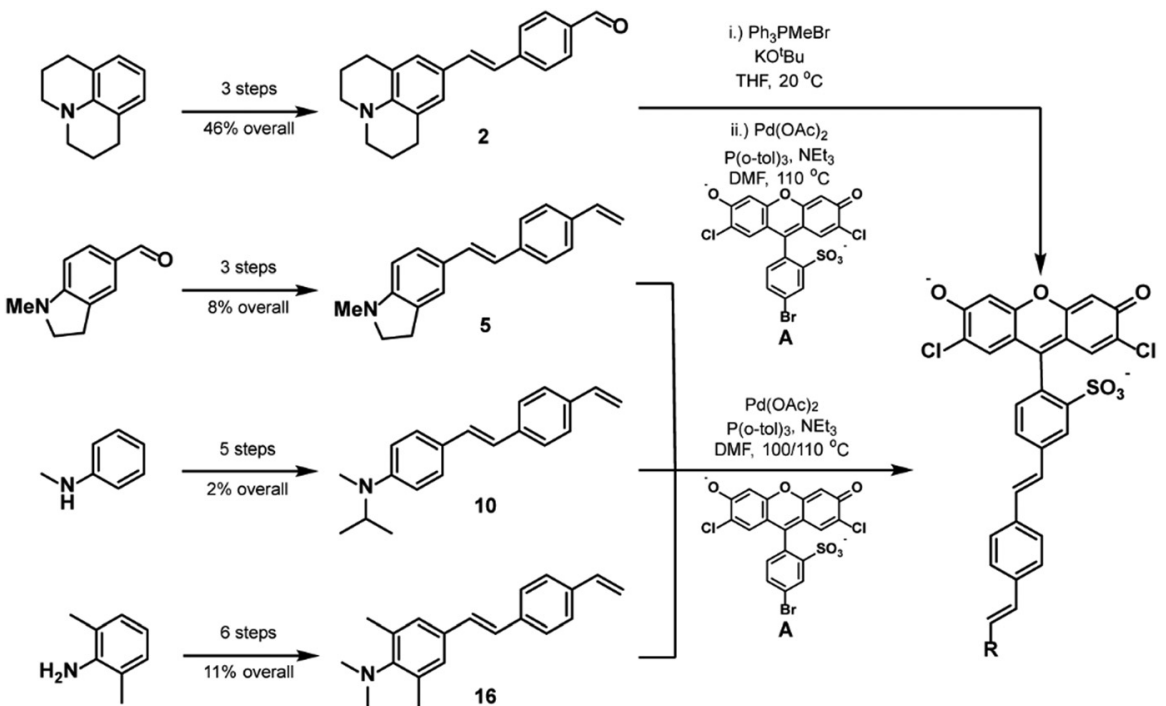

\begin{tabular}{|c|c|c|c|}
\hline$R=$ & Wire & VoltageFluor & $\%$ yield \\
\hline & 2 & 17 (JuloVF) & 7 \\
\hline & 5 & 18 (IndoVF) & 8 \\
\hline & 10 & 19 ('PrVF) & 7 \\
\hline
\end{tabular}

Scheme 2 Synthesis of new VF dye derivatives.

catalyzed cross coupling to provide molecular wire 2 in $52 \%$ yield over two steps (Scheme 2). This two-step sequence was performed on the same day to prevent oxidation of the electronrich styrene derived from julolidine 1 (Scheme S1, ESI $\dagger$ ). Another 2-step Wittig olefination and cross coupling with a sulfonated dichlorofluorescein (A) provided voltage indicator 17 (JuloVF, Scheme 2). Synthesis of $N$-methylindoline molecular wire 5 began with a Wittig olefination on commercially available $N$-methylindolinecarbaldehyde to provide 3 in $59 \%$ yield (Scheme S1, ESI $\dagger$ ). Pd-catalyzed cross coupling of 3 with 4-bromobenzaldehyde provided wire 4 in 34\% yield (Scheme S1, ESI $\dagger$ ). A final Wittig olefination gave 5 in 38\% yield (Scheme S1, ESI $\dagger$ ). Pd-Catalyzed cross coupling of 5 with a sulfonated dichlorofluorescein (A) provided voltage indicator 18 (IndoVF, Scheme 2).

$N$-Methyl- $N$-isopropyl molecular wire $\mathbf{1 0}$ was synthesized via the following route: reductive amination of acetone with $N$-methylaniline using $\mathrm{NaCNBH}_{3}$ gave $N$-isopropyl- $N$-methyl aniline 6 in 18\% yield (Scheme S2, ESI $\dagger$ ). In a similar sequence to the synthesis of JuloVF (17), Vilsmeier-Haack formylation with $\mathrm{POCl}_{3}$ in DMF gave aldehyde 7 (83\% yield) which was then converted to 8 through a Wittig olefination (56\% yield; Scheme S2, ESI $\dagger$ ). Olefin 8 was then converted to 9 (63\% yield) in a Pd-catalyzed cross coupling with 4-bromobenzaldehyde (Scheme S2, ESI $\dagger$ ). Subsequent Wittig olefination gave 10 in $44 \%$ yield, and a final Pd-catalyzed cross-coupling with a sulfonated dichlorofluorescein (A) provided voltage indicator 19 ( ${ }^{\mathrm{i}} \mathrm{PrVF}$, Scheme 2).

Synthesis of $N, N, 2,6$-tetramethylaniline wire 16 began with iodination of 2,6-dimethylaniline to give 11 in $67 \%$ yield (Scheme S2, ESI $\dagger$ ). Reductive amination of formaldehyde using $\mathrm{NaBH}_{4}$ gave $N, N, 2,6$-tetramethylaniline $\mathbf{1 2}$ in $95 \%$ yield (Scheme S2, ESI $\dagger$ ). Nucleophilic attack on dimethylformamide produced benzaldehyde $\mathbf{1 3}$ in $\mathbf{7 3 \%}$ yield (Scheme S2, ESI $\dagger$ ). Wittig olefination (14, 81\% yield), followed by a Pd-catalyzed cross coupling (15, $41 \%$ yield), and a second Wittig olefination a)

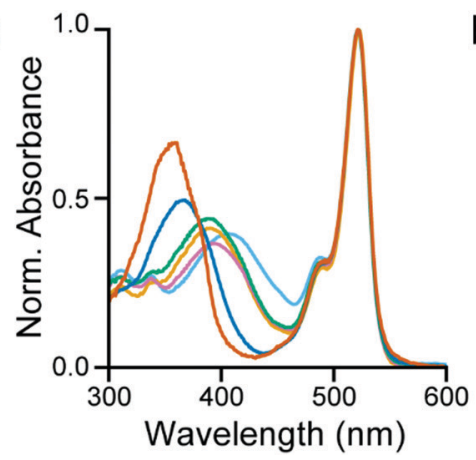

b)

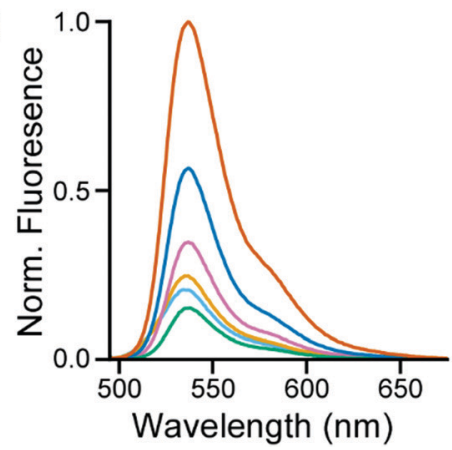

c)

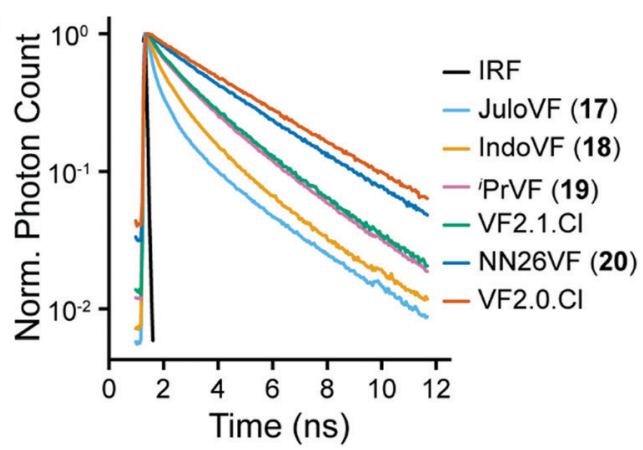

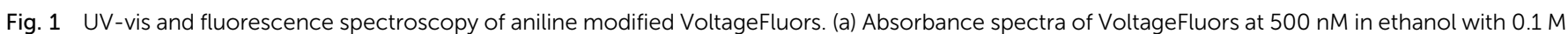

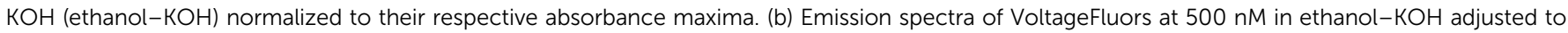

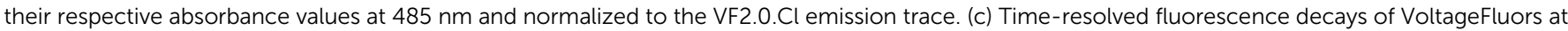

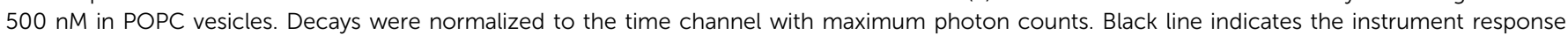
function (IRF). 
produced wire 16 (69\% yield) (Scheme S2, ESI $\dagger$ ). Pd-catalyzed cross coupling of $\mathbf{1 6}$ to a sulfonated dichlorofluorescein (A) yielded voltage indicator 20 (NN26VF, Scheme 2).

\section{Spectroscopic characterization of VF dyes}

To evaluate the degree of conjugation across the aniline series, we first examined the aniline modified VF library with UV-vis spectroscopy in ethanol with $0.1 \mathrm{M} \mathrm{KOH}$ (ethanol-KOH). Modification of the aniline altered the absorbance of the molecular wire region $(300-450 \mathrm{~nm})$ of these indicators but not the absorbance of the fluorescein chromophore region (450-550 nm) (Fig. 1a and Fig. S1, ESI $\dagger$ ). ${ }^{23,30}$ The wire region of JuloVF (17) displayed an absorbance maximum at $406 \mathrm{~nm}$, a red-shift of $17 \mathrm{~nm}$ relative to the wire region of $\mathrm{VF} 2.1 . \mathrm{Cl}$ (Table 1). In contrast, the wire region of NN26VF (20) displayed an absorbance maximum at $368 \mathrm{~nm}$, a blue-shift of $21 \mathrm{~nm}$ relative to the wire region of $\mathrm{VF} 2.1$.Cl (Table 1 ). For comparison, the wire region of $\mathrm{VF} 2.0 . \mathrm{Cl}$, which completely lacks an aniline, displayed an absorbance maximum at $360 \mathrm{~nm}$ (Table 1). The wire regions of IndoVF (18) and ${ }^{\mathrm{i}} \operatorname{PrVF}$ (19) displayed absorbance maxima very close to VF2.1.Cl (Table 1 ).

The trend observed in wire absorbance maxima is reproduced in the ${ }^{13} \mathrm{C}$ NMR chemical shifts of the carbon para to the aniline nitrogen. Steric inhibition of conjugation ${ }^{33,34}$ (such as in NN26VF, 20) results in a downfield shift, while increased conjugation moves chemical shifts upfield, as a result of increased shielding. This trend holds for reported anilines (Fig. S2a, ESI $\dagger)^{34-40}$ and for the benzaldehyde-derived anilines reported in this manuscript (Fig. S2b, ESI $\dagger$ ). ${ }^{13} \mathrm{C}$ NMR assignment for the unsymmetrical aniline, 1-methylindoline-5carbaldehyde, was guided by 2D NMR (Spectra S45 and S46).

Finally, we also performed calculations on the parent anilines of the VF dye series at the B3LYP-D3(BJ)/6-311G* level of theory. HOMO energy levels were calculated for the geometry optimized anilines and match the trend in ${ }^{13} \mathrm{C}$ NMR chemical shifts: electron-rich molecular wires with highly conjugated anilines, such as JuloVF (17) have the highest HOMO energy levels, while NN26VF (20) has the lowest (Fig. S3a, ESI $\dagger$ ). Taken together, this data suggests that VF dyes will have more negative values of $\Delta G_{\mathrm{PeT}}$, and therefore increased rates of PeT, ${ }^{41}$ as aniline conjugation to the molecular wire increases and the
HOMO and ionization potential of the molecular wire increases. $^{16}$

To confirm that the observed spectroscopic trends are a result of varying degrees of aniline nitrogen conjugation to the aromatic $\pi$ system, we examined the absorbance spectra of the VF dye series in aqueous buffers of varying $\mathrm{pH}$ values. Protonation of the aniline in acidic buffer should result in a hypsochromic shift in the wire absorbance, as the aniline lone pair is no longer delocalized throughout the wire. Indeed, at low $\mathrm{pH}$, we observe wire absorbance maxima around 360-365 nm for all VF dyes (Fig. S4, ESI $\dagger$ ), eliminating spectral differences between dyes which were seen in basic conditions. Therefore, we reason that the differences in wire absorbance maxima in basic buffer result from differences in the degree of conjugation of the aniline lone pair with the rest of the molecular wire. Two dyes, ${ }^{\mathrm{i}} \mathrm{PrVF}$ (19) and NN26VF (20), displayed hypsochromic shifts at $\mathrm{pH} 7.5$, whereas the remaining $\mathrm{VF}$ dyes (JuloVF, IndoVF, and VF2.1.Cl) displayed equivalent shifts in molecular wire absorbance at pH 5 (Fig. S4, ESI $\dagger$ ).

Next, we measured the emission, quantum yield $\left(\Phi_{\mathrm{fl}}\right)$, and fluorescence lifetime $\left(\tau_{\mathrm{fl}}\right)$ of the VF series in solution. We observed consistent shapes of the emission spectra from the shared dichlorofluorescein chromophore, but there were clear differences in the $\Phi_{\mathrm{fl}}$ and $\tau_{\mathrm{fl}}$ (Fig. 1b, c and Fig. S5, ESI $\dagger$ ). VF2.0.Cl had the highest $\Phi_{\mathrm{fl}}$ in ethanol-KOH, followed by NN26VF (20), i PrVF (19), IndoVF (18), JuloVF (17), and VF2.1.Cl respectively (Table 1 ). This trend was replicated in the $\tau_{\mathrm{fl}}$ measured in ethanol-KOH (Table 1).

\section{Cellular characterization of VoltageFluors}

Having performed initial characterization across our series of aniline donors in vitro, we next examined VF dye performance in cells. VF2.0.Cl was the brightest indicator in HEK cells, followed by VF2.1.Cl, NN26VF (20), ${ }^{\mathrm{i}} \mathrm{PrVF}$ (19), IndoVF (18) and JuloVF (17), which was by far the dimmest (Table 1 and Fig. S6, ESI $\dagger$ ). Because JuloVF (17) was so dim, functional cellular characterizations of JuloVF (17) were carried out at $500 \mathrm{nM}$ dye loading to generate sufficient signal for analysis.

To measure voltage sensitivity of these indicators, we turned to whole-cell voltage clamp in HEK293T cells stained with dye. By recording the changes in fluorescence intensity with

Table 1 Properties of VoltageFluor dyes

\begin{tabular}{|c|c|c|c|c|c|c|c|c|c|}
\hline Dye & $\begin{array}{c}\text { Wire } \lambda_{\text {abs }}{ }^{a} \\
(\mathrm{~nm})\end{array}$ & $\Phi_{\mathrm{fl}}{ }^{a}$ & $\tau_{\mathrm{fl}}^{a}$ & $\tau_{\mathrm{fl}}^{b}$ & $\begin{array}{l}\text { Rel. brightness }^{c} \\
\text { (HEK293T) }\end{array}$ & $\% \Delta F / F^{e}$ & $\mathrm{SNR}^{e}$ & $\Delta \tau_{\mathrm{fl}}$ per $\mathrm{mV}(\mathrm{ps})$ & $\tau_{\mathrm{fl}}$ at $0 \mathrm{mV}(\mathrm{ns})$ \\
\hline JuloVF (17) & 406 & 0.10 & $0.63 \pm 0.08$ & $0.81 \pm 0.05$ & $\begin{array}{l}0.021 \pm 0.003 \\
0.037 \pm 0.004^{d}\end{array}$ & $34 \pm 6^{d}$ & $26 \pm 2$ & $0.73 \pm 0.03^{d}$ & $0.31 \pm 0.01^{d}$ \\
\hline${ }^{\mathrm{i}} \operatorname{PrVF}(\mathbf{1 9})$ & 392 & 0.28 & $1.04 \pm 0.04$ & $1.67 \pm 0.03$ & $0.29 \pm 0.03$ & $34 \pm 2$ & $125 \pm 2$ & $2.97 \pm 0.04$ & $1.28 \pm 0.01$ \\
\hline VF2.1.Cl & 389 & 0.12 & $0.53 \pm 0.02$ & $1.58 \pm 0.05$ & $1.0 \pm 0.1$ & $26 \pm 3$ & $200 \pm 10$ & $3.05 \pm 0.08$ & $1.57 \pm 0.03$ \\
\hline NN26VF (20) & 368 & 0.44 & $1.66 \pm 0.03$ & $2.55 \pm 0.06$ & $0.44 \pm 0.05$ & $2.2 \pm 0.1$ & $8.6 \pm 0.2$ & $0.50 \pm 0.02$ & $3.32 \pm 0.01$ \\
\hline
\end{tabular}

Solution phase $\tau_{\mathrm{fl}}$ measurements were taken at $500 \mathrm{nM}$ dye. Cellular measurements were conducted at $300 \mathrm{nM}$ dye loading unless otherwise noted. ${ }^{a}$ In ethanol-KOH. ${ }^{b}$ In POPC. ${ }^{c}$ All brightness values are relative to VF2.1.Cl (1.0 $\times$ brightness), and were calculated as the difference between cell signal and the surrounding background (see Fig. S6, ESI for additional details). ${ }^{d}$ Acquired at $500 \mathrm{nM}$ dye loading on account of the low cellular brightness of JuloVF (17). ${ }^{e}$ Per $100 \mathrm{mV}$ in HEK293T cells. Data are mean \pm S.E.M. for $n=3-8$ different cells. 

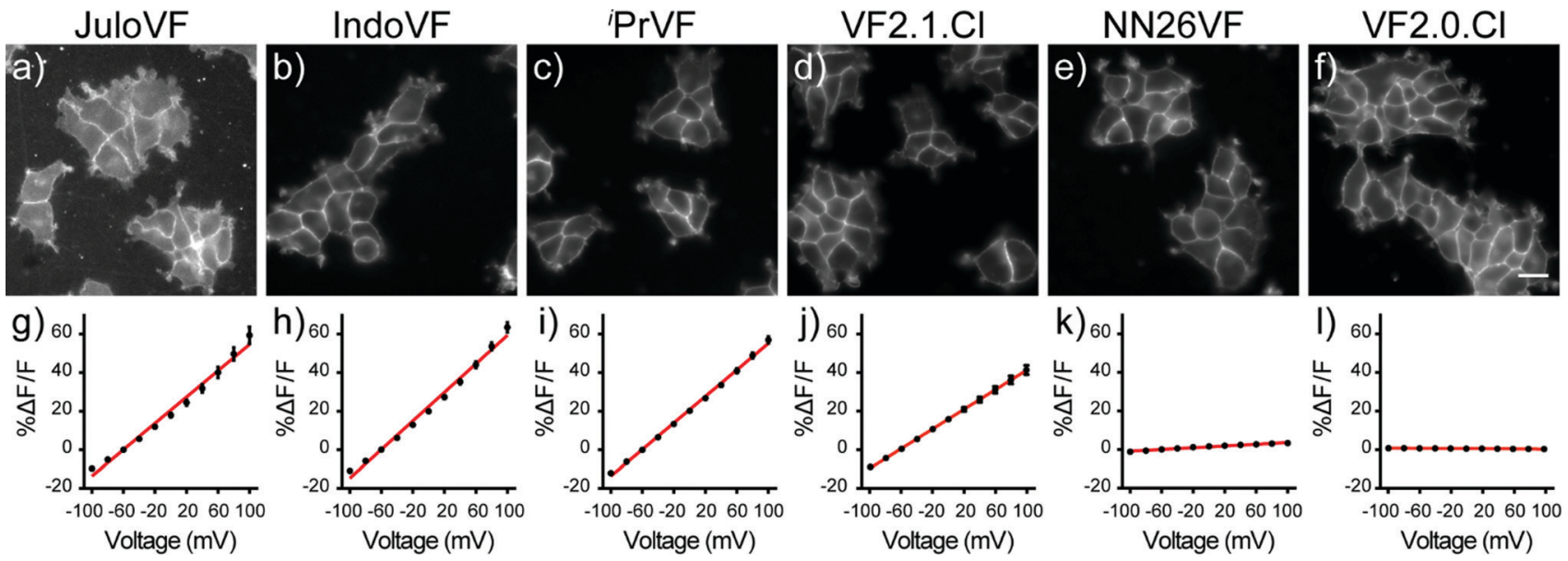

Fig. 2 Relative voltage sensitivity and relative brightness of VoltageFluor derivatives in HEK cells. Widefield fluorescence images of HEK cells stained with $500 \mathrm{nM}$ (a) JuloVF or $300 \mathrm{nM}$ (b) IndoVF, (c) 'PrVF, (d) VF2.1.Cl, (e) NN26VF, or (f) VF2.0.Cl. In order to show the membrane localization of each indicator, images are not scaled to the same grey values. Scale bar is $20 \mu \mathrm{m}$. Plots of relative change in fluorescence intensity $(\% \Delta F / F) v s$. membrane potential (in millivolts) for (g) JuloVF, (h) IndoVF, (i) i PrVF, (j) VF2.1.Cl, (k) NN26VF, and (l) VF2.0.Cl. Cellular measurements were conducted at $300 \mathrm{nM}$ dye loading except for experiments with JuloVF which were conducted at $500 \mathrm{nM}$ dye loading. Data are mean fluorescence values \pm S.E.M. for $n=3-8$ different cells. When not visible, error bars are smaller than the data points.

epifluorescence microscopy when voltage steps from 100 to $-100 \mathrm{mV}$ were applied (in $20 \mathrm{mV}$ increments), we observed a dramatic difference in voltage sensitivity based on the identity of the aniline donor. As previously reported, VF2.1.Cl has modest relative sensitivity to $V_{\text {mem }}$ changes $(26 \% \Delta F / F$ per $100 \mathrm{mV}$, Fig. $2 \mathrm{j}$, Fig. S6, ESI $\dagger$ and Table 1), but three of the newly synthesized indicators have higher relative voltage sensitivies $(\Delta F / F$ per $100 \mathrm{mV}$ ) than VF2.1.Cl. Voltage indicators with ring-fused anilines, IndoVF (18) and JuloVF (17), displayed larger fractional fluorescence intensity responses to voltage changes $37 \%$ and $34 \% \Delta F / F$ per $100 \mathrm{mV}$, respectively) (Fig. 2g, h, Fig. S6, ESI $\dagger$ and Table 1). ${ }^{\mathrm{i}} \mathrm{PrVF}$ (19) also had a larger relative response to $V_{\text {mem }}$ changes (34\% $\Delta F / F$ per $100 \mathrm{mV}$, Fig. 2i, Fig. S6, ESI $\dagger$ and Table 1) than VF2.1.Cl. As previously reported, indicators lacking an aniline donor, like VF2.0.Cl, possess little to no relative sensitivity $(-0.2 \% \Delta F / F$ per $100 \mathrm{mV}$, Fig. 2l, Fig. S6, ESI $\dagger$ and Table 1). When methyl groups are added ortho to the aniline nitrogen (NN26VF, 20), relative voltage sensitivity drops to $2.2 \% \Delta F / F$ per $100 \mathrm{mV}$ (Fig. 2k, Fig. S6 (ESI $\dagger$ ) and Table 1), an order of magnitude lower than VF2.1.Cl.

After examining our VF dye series with fluorescence intensity imaging, we then analyzed the series with fluorescence lifetime imaging microscopy (FLIM, Fig. 3). With simultaneous FLIM and whole cell voltage clamp electrophysiology, we record the lifetime in cells at different $V_{\text {mem }}$ and then calculate a line of best fit for each individual cell's lifetime- $V_{\text {mem }}$ calibration (Fig. S7-S12, ESI $\dagger$ ). From these lifetime- $V_{\text {mem }}$ lines of best fit, we extract the absolute sensitivity of fluorescence lifetime to $V_{\text {mem }}\left(\Delta \tau_{\mathrm{fl}}\right.$ per $\left.\mathrm{mV}\right)$ and the baseline lifetime $\left(\tau_{\mathrm{fl}}\right.$ at $\left.0 \mathrm{mV}\right)$ in HEK293T cells (Fig. 3 and Fig. S7-S12, ESI $\dagger$ ). ${ }^{31}$

Critically, all of the new aniline modified VFs show $V_{\text {mem }}$ sensitive fluorescence lifetimes, with absolute voltage sensitivities ranging from 0.50 to $2.97 \mathrm{ps} \mathrm{mV}^{-1}$ (Fig. 3g) and baseline lifetimes from 0.31 to $3.32 \mathrm{~ns}$ (Fig. 3 h and Table 1 ). Dimmer VFs such as JuloVF (17) and IndoVF (18) have a high relative $V_{\text {mem }}$ sensitivity $(\% \Delta F / F$ or $\% \Delta \tau / \tau$, Fig. 2 and Fig. S13, ESI $\dagger)$, but a lower absolute $\Delta \tau_{\text {fl }}$ per $\mathrm{mV}$ (Fig. $3 \mathrm{~g}$ ). We observe the highest absolute voltage sensitivities for newly synthesized ${ }^{\mathrm{i}} \operatorname{PrVF}$ (19) and VF2.1.Cl, which display intermediate baseline lifetimes close to $1.5 \mathrm{~ns}$ at $0 \mathrm{mV}$ (Fig. 3h). Very long lifetimes, such as those in NN26VF (20), are associated with both low relative (Fig. 2k) and absolute (Fig. 3g) voltage sensitivities.

To construct $\tau_{\mathrm{fl}}-V_{\text {mem }}$ calibrations, we selected exponential models for the new VFs based on minimization of reduced chi squared without overfitting (Fig. S14, S15 and Table S4, ESI $\dagger$ ). VFs dyes with shorter lifetimes (e.g. JuloVF [17], IndoVF [18]), could not be well described with fewer than 3 exponential decay components, but use of a 3-component decay model for the other VF indicators resulted in overfitting.

All lifetime data were acquired at $300 \mathrm{nM}$ dye concentration. At this concentration, all dyes displayed concentrationindependent $\tau_{\mathrm{fl}}$ (Fig. S16, ESI $\dagger$ ) but retained suffient brightness for cellular imaging (Table S1, ESI $\dagger$ ). We found that the voltage sensitivities of VF2.1.Cl and VF2.0.Cl were not substantially different when loading with $300 \mathrm{nM}$ dye instead of the optimized concentration (100 nM) used in previous fluorescence lifetime studies (Tables S2 and S3, ESI $\dagger$ ). ${ }^{31}$ JuloVF (17) was used at $500 \mathrm{nM}$ because of its very low brightness in cells. As a result, fluorescence lifetime data for JuloVF (17) in cells may contain contributions from autofluorescence and concentration quenching. ${ }^{31,42}$

We compared values of fluorescence lifetime for VF dyes measured in cells (Fig. 3, Fig. S7-S12, ESI $\dagger$ and Table 1) to the values of $\tau_{\mathrm{fl}}$ we obtained from in vitro solution measurements in EtOH-KOH (Fig. S5c, ESI $\dagger$ and Table 1). We found that the $\tau_{\mathrm{fl}}$ in EtOH was only partially correlated with the $\tau_{\mathrm{fl}}$ at $0 \mathrm{mV}$ in cells, with some probes showing considerable discrepancies (Fig. S17a, ESI, $\dagger r^{2}=0.70$ ). Correlation between $\tau_{\mathrm{fl}}$ in EtOH and 
a)
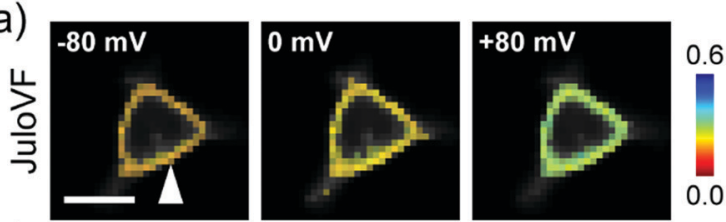

b)
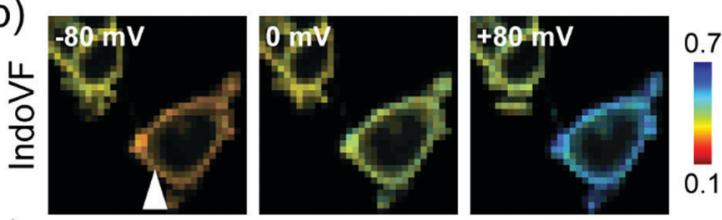

c)
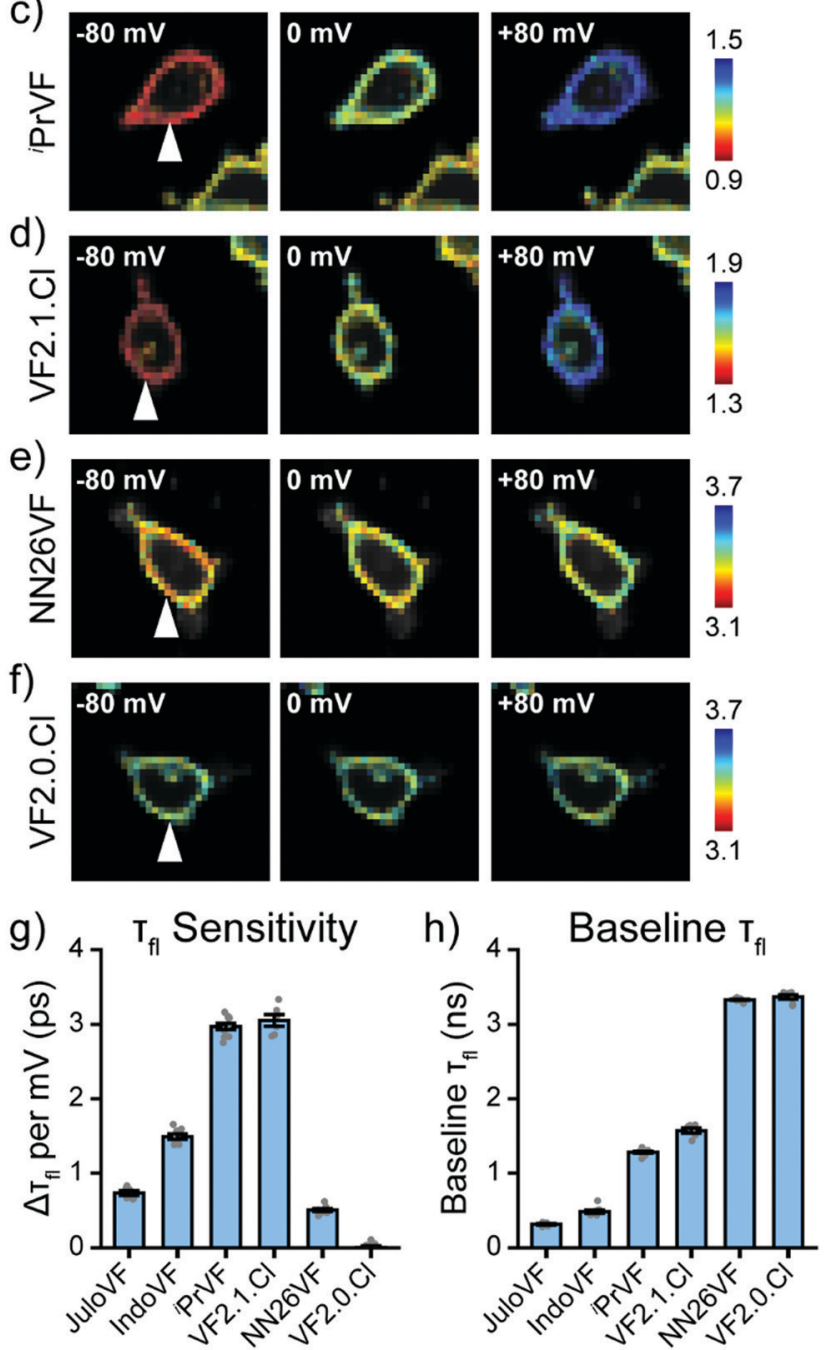

Fig. 3 Fluorescence lifetime captures absolute sensitivity of VFs. (a-f) Lifetime-intensity overlay images for HEK293T cells at different $V_{\text {mem }}$ stained with JuloVF, IndoVF, iPrVF, VF2.1.Cl, NN26VF, and VF2.0.Cl, respectively (300 $\mathrm{nM}$ dye was used for all except JuloVF at $500 \mathrm{nM}$ ). $V_{\text {mem }}$ was held at the indicated value with whole cell voltage clamp electrophysiology. Lifetimes are scaled across the same lifetime range (0.6 ns) with different start and ending values. White arrow indicates voltageclamped cell. Scale bar represents $20 \mu \mathrm{m}$. (g) Absolute lifetime sensitivity $\left(\Delta \tau_{\mathrm{fl}}\right.$ per $\mathrm{mV}$ ) for the aniline modified VF library. Gray points represent measurements from individual cells. (h) Baseline lifetime ( $y$-intercept/lifetime at $0 \mathrm{mV}$ ) from the same lines of best fit as in (g). Data are mean \pm SEM for the following number of patched cells: JuloVF 6, IndoVF 8, 'PrVF 10, VF2.1.Cl 6, NN26VF 8, VF2.0.Cl 7. the molecular wire absorbance $\lambda_{\max }$ is similar (Fig. S17b, ESI, $\dagger$ $\left.r^{2}=0.75\right)$. We hypothesized that differences in indicator environment between EtOH-KOH and the cell membrane could account for these discrepancies. To better model the lipid environment of the plasma membrane in vitro, we measured $\tau_{\mathrm{fl}}$ in vesicles of 1-palmitoyl-2-oleoyl-glycero-3-phosphocholine 16:0-18:1 PC (POPC) (Table 1 and Fig. 1c).

We observed stronger correlation between in vitro $\tau_{\mathrm{fl}}$ values measured in POPC vesicles and those measured in cells at $0 \mathrm{mV}$ (Fig. S17c, ESI, $\uparrow r^{2}=0.95$ ) than we had with $\tau_{\mathrm{fl}}$ in EtOH-KOH (Fig. S17a, ESI $\dagger$ ). The values of $\tau_{\mathrm{fl}}$ in POPC closely follow the shifts in the molecular wire $\lambda_{\max }$ (Fig. S17d, ESI, $\uparrow r^{2}=0.91$ ). Together, these data suggest that POPC vesicles are a good proxy for cellular membranes, although the exact values of probe $\tau_{\mathrm{fl}}$ differ between POPC and the plasma membrane.

\section{VoltageFluors in electrically excitable cells}

We evaluated the aniline-modified VFs with the three highest absolute voltage sensitivities (IndoVF [18], ${ }^{\mathrm{i}} \operatorname{PrVF}$ [19], and VF2.1.Cl) for their ability to monitor electrical activity in human induced pluripotent stem cell derived cardiomyocytes (hiPSC$\mathrm{CMs}$ ) and dissociated rat hippocampal neurons. All three indicators faithfully recorded action potential (AP) waveforms in spontaneously contracting monolayers of cardiomyocytes (Fig. 4a-f) and evoked action potentials in dissociated rat hippocampal neurons (Fig. 4h-m). The average signal-tonoise ratio (SNR) of activity recordings in cardiomyocyte monolayers was high in all cases, with ${ }^{\mathrm{i}} \operatorname{PrVF}$ (19) and VF2.1.Cl having the highest SNR values: in excess of $400: 1$ (Fig. 4g, Fig. S18a-h, ESI $\dagger$ and Table 2). IndoVF (18) displays lower SNR values $(140: 1)$ but is still capable of reporting cardiac action potential kinetics (Fig. 4f, g and Fig. S18i, ESI $\dagger$ ). In neurons, VF2.1.Cl exhibits the highest average SNR $(13: 1)$ for evoked activity recordings (Fig. 4n, Fig. S19, ESI $\dagger$ and Table 2). The other two VF dyes had lower SNR values, 9.7:1 for ${ }^{\mathrm{i}} \operatorname{PrVF}(19)$ and 5.6:1 for IndoVF (18) (Fig. 4n, Fig. S19, ESI $\dagger$ and Table 2).

We also investigated the phototoxicity and photostability of these three derivatives, as we previously observed differences in the phototoxicity of PeT-based voltage indicators with different wire structures. ${ }^{29}$ We compared the phototoxicity of ${ }^{i} \operatorname{PrVF}$ (19) and IndoVF (18) to VF2.1.Cl during prolonged measurements of activity in iPSC-CM monolayers. With all three sensors, we were able to record APs without alterations to the AP waveform for up to 4 minutes (Fig. S20a-d, ESI $\dagger$ ). IndoVF (18) appeared slightly less phototoxic than ${ }^{\mathrm{i}} \operatorname{PrVF}$ (19) or VF2.1.Cl. AP kinetics reported by IndoVF (18) remain unchanged for approximately 6 minutes of continous illumination in tissue (Fig. S20d-f, ESI $\dagger$ ). The initial photobleaching rates for all three indicators are similar in HEK293T cells, iPSCCMs, and dissociated rat hippocampal neurons (Fig. S21, ESI $\dagger$ ). These experiments establish that modifying the aniline conformation has minimal effect on probe photobleaching and phototoxicity, and IndoVF (18), ${ }^{\mathrm{i}} \operatorname{PrVF}$ (19), and VF2.1.Cl are all capable of reporting on cardiac and neuronal electrophysiology with high SNR. 

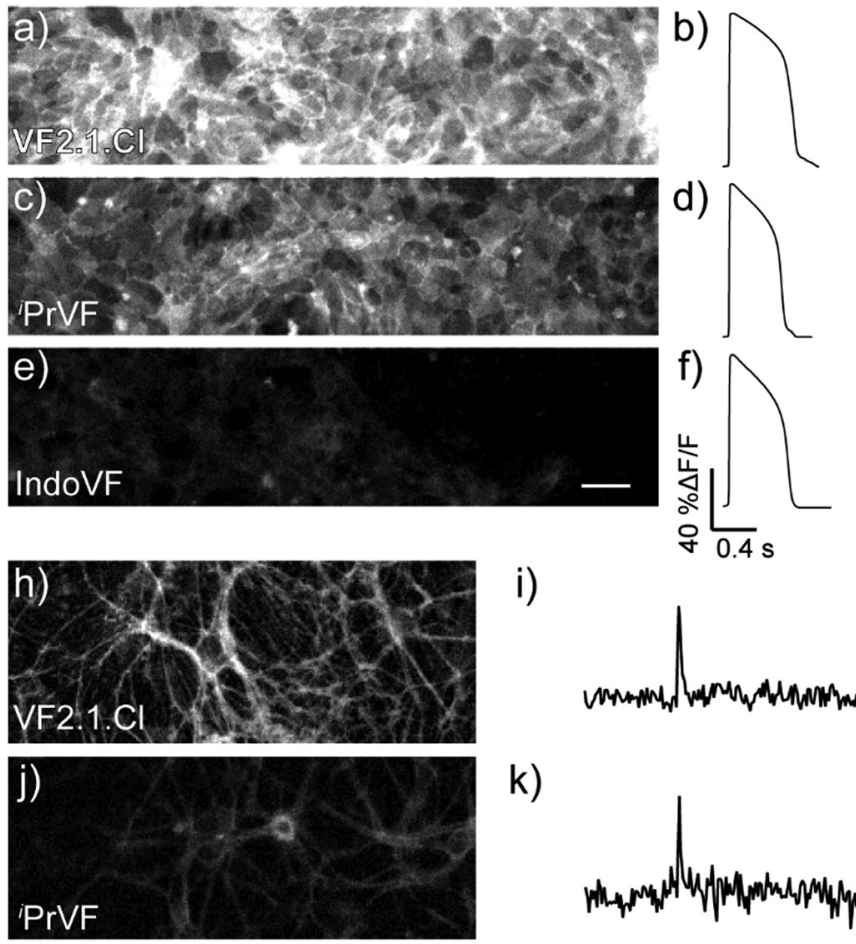

d)

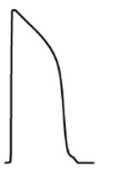

f)
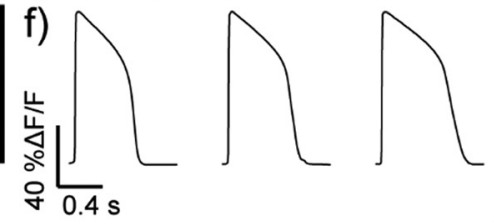

$\int^{g}$
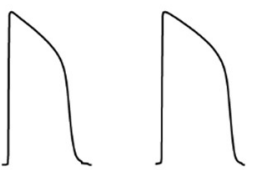

I)

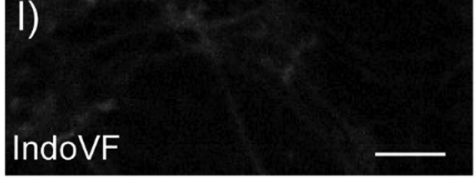

i)

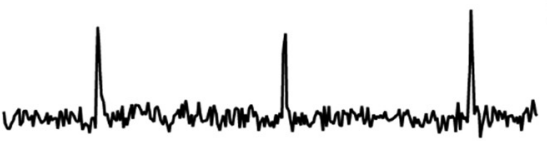

k)

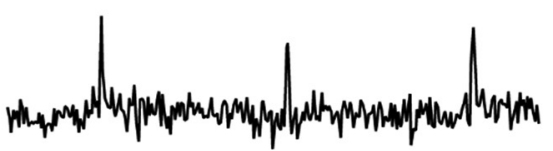

n) (VF2.1.Cl), 46 (IndoVF), 58 ('PrVF). Scale bars are $50 \mu \mathrm{m}$.
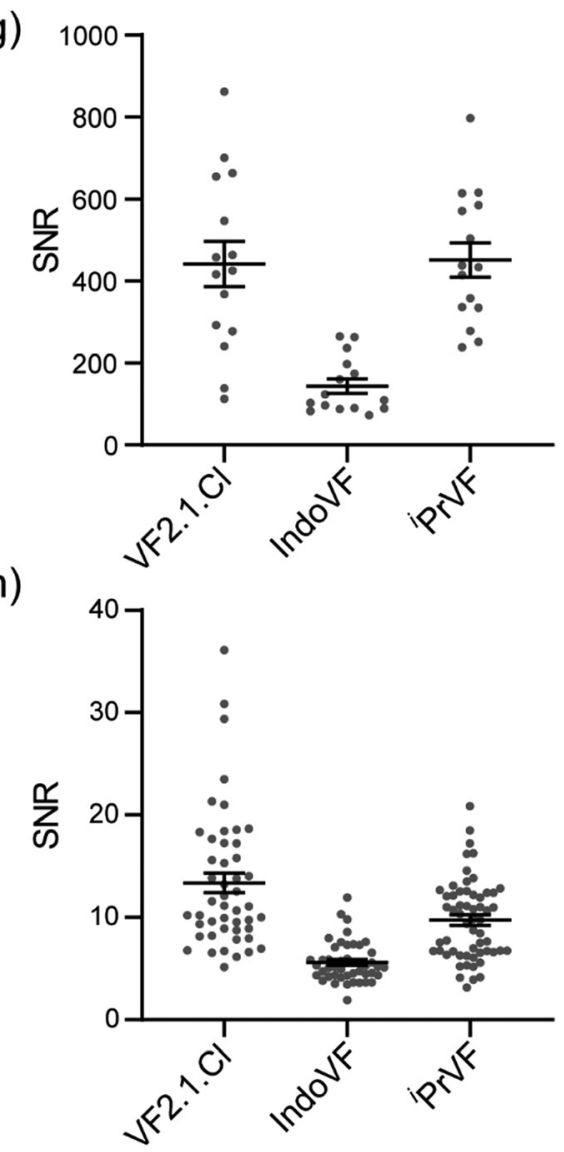

Fig. 4 VF performance in excitable cells. Representative field of view (FOV) used for recordings of evoked electrical activity in iPSC-CMs loaded with $300 \mathrm{nM}$ (a) VF2.1.Cl, (c) 'PrVF, and (e) IndoVF. Average action potentials from three ten second acquisitions of iPSC-CMs loaded with $300 \mathrm{nM}$ (b) VF2.1.Cl, (d) 'PrVF, and (f) IndoVF. (g) Signal-to noise ratio (SNR) plot of spontaneous action potentials, where dots are the SNR for a single acquisition. Bars are mean \pm SEM. $N=15$ traces. Representative field of view (FOV) used for recordings of evoked electrical activity in dissociated rat hippocampal neurons loaded with $300 \mathrm{nM}$ (h) VF2.1.Cl, (j) 'PrVF, and (l) IndoVF. Action potentials evoked from a single neuron loaded with $300 \mathrm{nM}$ (i) VF2.1.Cl, (k) 'PrVF, and (m) IndoVF. (n) SNR plot of evoked action potentials. Points are the SNR of a single action potential from a unique neuron. Bars are mean \pm SEM. $N=48$

Table 2 SNR values in cellular voltage measurements

\begin{tabular}{llc}
\hline VoltageFluor & hiPSC-CM & Neuron \\
\hline IndoVF (18) & $140 \pm 4$ & $5.6 \pm 0.3$ \\
${ }^{i}$ PrVF (19) & $450 \pm 10$ & $9.7 \pm 0.5$ \\
VF2.1.Cl & $440 \pm 10$ & $13 \pm 1$
\end{tabular}

Data are mean SNR \pm SEM. All data were measured at $300 \mathrm{nM}$.

\section{Discussion}

We hypothesized that VF dyes containing aniline groups with greater conjugation to the aromatic $\pi$ system would experience faster rates of PeT, reducing $\Phi_{\mathrm{fl}}$ and $\tau_{\mathrm{fl}}$. Increased conjugation of the aniline nitrogen with the molecular wire $\pi$ system increases the HOMO energy level (Fig. S3, ESI $\dagger$ ), increasing the ionization potential of the molecular wire/aniline donor. This increased ionization potential makes the Gibbs free energy for PeT $\left(\Delta G_{\mathrm{PeT}}\right)$ more negative and, according to the Rehm-Weller equation, ${ }^{41}$ increases the rate of PeT. Conversely,
VF dyes containing aniline groups with reduced conjugation to the aromatic $\pi$ system would experience slower rates of PeT, increasing $\Phi_{\mathrm{fl}}$ and $\tau_{\mathrm{fl}}$. Using this framework, we modulated baseline $\tau_{\mathrm{fl}}$ values, signal-to-noise ratios, relative voltage sensitivities, and absolute voltage sensitivities in VF dyes. Tuning these values allowed us to identify dyes with optimal performance in action potential detection.

\section{Extent of conjugation alters PeT}

Our initial spectroscopic characterizations demonstrated that the identity of the aniline donor group affected the extent of conjugation in the molecular wire in VF dyes. The molecular wire region of JuloVF (17), which contains an annulated aniline, displayed a red-shifted absorbance maximum relative to VF2.1.Cl (Fig. 1a). In contrast, NN26VF (20) contains an aniline with little conjugation to the aromatic $\pi$ system and displayed a blue-shifted absorbance maximum relative to VF2.1.Cl (Fig. 1a). We believe these absorbance shifts are the result of the degree of conjugation of the aniline nitrogen because protonation of 
the nitrogen in acidic buffer ablates the spectral differences observed in basic buffer (Fig. S4, ESI $\dagger$ ).

Our observations in ${ }^{13} \mathrm{C}$ NMR spectra and calculated HOMO energy levels of molecular wire precursors are consistent with the UV-vis absorbance spectra of the VF series. The carbon para to the aniline in molecular wire precursors displays an increasingly upfield ${ }^{13} \mathrm{C}$ NMR shift as aniline conjugation increases due to increased shielding (Fig. S2, ESI $\dagger$ ), matching literature values for reported dialkylanilines. ${ }^{40,43-49}$ Similarily, the calculated HOMO energy level for molecular wire precursors increases as aniline conjugation increases across the VF dye series (Fig. S3, ESI $\dagger$ ). The higher HOMO energy levels and oxidation potential of more conjugated molecular wires increases the driving force for PeT.

The trend in $\Phi_{\mathrm{fl}}$ and $\tau_{\mathrm{fl}}$ measured in ethanol-KOH is consistent with our hypothesis that the extent of conjugation of the aniline group affects PeT (Table 1), although VF2.1.Cl has lower-than-expected $\Phi_{\mathrm{fl}}$ and $\tau_{\mathrm{fl}}$ values in EtOH-KOH. Because we were concerned that solvent effects may be altering our results, we compared the lifetime in EtOH to that in POPC vesicles, which more closely mimic the lipid membrane (Fig. S17, ESI $\dagger$ ). In POPC vesicles, the $\tau_{\mathrm{fl}}$ values of all dyes track more closely with the molecular wire $\lambda_{\text {max }}$ (Fig. S17d, ESI $\dagger$ ). In particular, in POPC, VF2.1.Cl now displays a higher, intermediate value of $\tau_{\mathrm{fl}}$ which more closely matches values in cells and the absorbance spectroscopy data (Fig. S17, ESI $\dagger$ and Table 1). The $\tau_{\mathrm{fl}}$ of all aniline-containing VFs was longer in POPC than in $\mathrm{EtOH}-\mathrm{KOH}$; this may be attributable to the effects of $\mathrm{pH}$ and solvent dielectric constant on electron transfer rate. ${ }^{50}$ Minimal, if any, concentration dependence was seen for $\tau_{\mathrm{fl}}$ in both EtOH$\mathrm{KOH}$ and POPC, suggesting that concentration quenching is not responsible for these trends (Fig. S5, ESI $\dagger$ ).

\section{Fluorescence lifetime predicts probe performance}

Moving from in vitro characterization to cellular experiments, we found that the fluorescence intensity of VFs in HEK293T cells does not correlate strongly with the fluorescence lifetime (Fig. 5a). This result emphasizes that caution should be used in interpreting probe brightness in cells in terms of fundamental photophysics. Fluorescence intensity, and metrics that rely solely on fluorescence intensity, like SNR, are confounded by dye concentration in the membrane, excitation intensity, changes in detector gain or integration time, and differences in excitation and emission filter sets. These variables can profoundly alter the values of SNR, meaning that comparisons of SNR must be peformed under identical conditions, making SNR a less portable metric for comparing across large indicator libraries. Furthermore, observation of high or low SNR offers very little information about the mechanistic reasons for good or poor indicator performance. On the other hand, FLIM measurements of $\tau_{\mathrm{fl}}$, although more technically challenging to implement, is not strongly influenced by the variables listed above, and therefore, provides a means to understand factors that contribute to low SNR.

Examining the voltage dependence of $\tau_{\mathrm{fl}}$, we noticed a parabolic relationship between the absolute voltage sensitivity
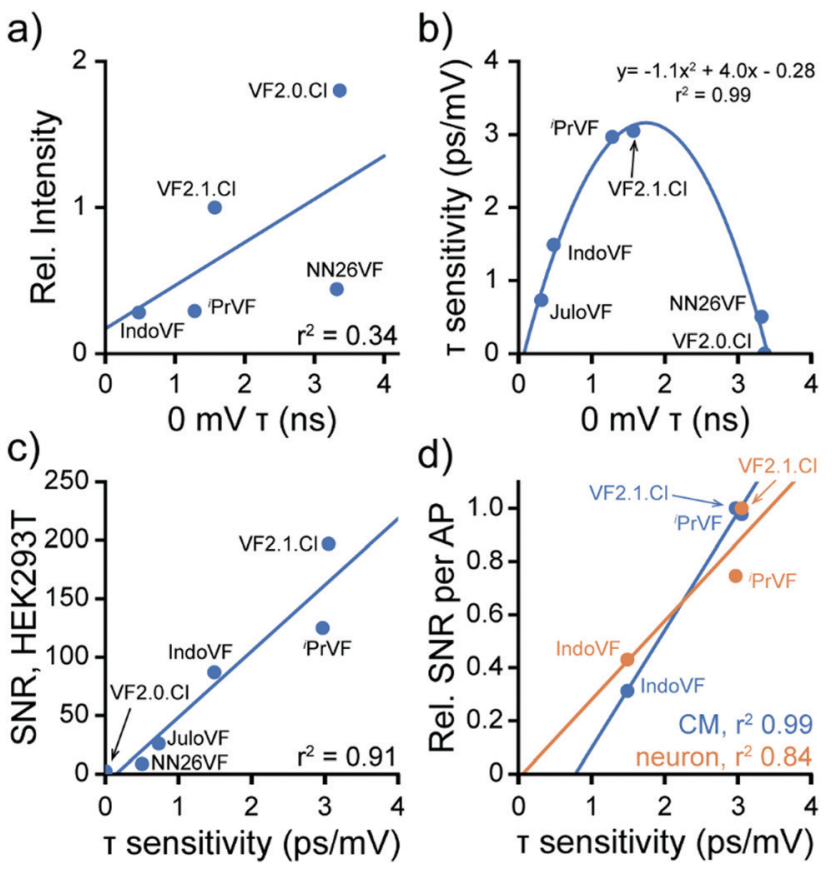

Fig. 5 Fluorescence lifetime change dictates VF performance. Data are aggregated from Fig. 2, 3 and 4 to highlight properties of the VF library. (a) $0 \mathrm{mV}$ lifetime and fluorescence intensity of VFs at $300 \mathrm{nM}$ are only somewhat correlated in HEK cells. JuloVF is omitted here because no lifetime data were taken at $300 \mathrm{nM}$. Line of best fit (blue): $y=1.2 x+1.1$, $r^{2}=0.34$. (b) Relationship between baseline lifetime at $0 \mathrm{mV}$ and voltage sensitivity in fluorescence lifetime $\left(\Delta \tau_{\mathrm{fl}}\right.$ per $\left.\mathrm{mV}\right)$ for $\mathrm{VF}$ dyes in HEK293T cells. Line indicates a parabola fit to the data. (c) Correlation between $\Delta \tau_{\mathrm{fl}}$ per $\mathrm{mV}$ and the signal to noise ratio (SNR) of a $100 \mathrm{mV} V_{\text {mem }}$ step ( -60 to $+40 \mathrm{mV}$ ) in HEK293T. Line of best fit (blue): $y=56.7 x-8.2, r^{2}=0.91$. (d) Correlation between $\Delta \tau_{\mathrm{fl}}$ per $\mathrm{mV}$ and the SNR for detection of spontaneous APs in cardiomyocytes (CM, blue) or evoked APs in neurons (orange). The SNR for each probe in each system (Tables 1 and 2) was normalized to the maximum SNR seen in that system for ease of comparison. CM line of best fit (blue): $y=0.44 x-0.35, r^{2}=0.99$; neuron line of best fit (orange): $y=0.30 x-0.02, r^{2}=0.84$. For $(a-d)$, each point represents the mean across all measurements of each type made for each dye; error bars are omitted for clarity. All dyes were used at $300 \mathrm{nM}$, except for JuloVF, where $500 \mathrm{nM}$ was used.

$\left(\Delta \tau_{\mathrm{fl}}\right.$ per $\left.\mathrm{mV}\right)$ and baseline $\tau_{\mathrm{fl}}$ of the VFs (Fig. $\left.5 \mathrm{~b}\right)$. Because all of the VFs in this study possess identical dichlorofluorescein chromophores and have the same extinction coefficient, the baseline $\tau_{\mathrm{fl}}$ in patch-clamped HEK293T cells reflects differences in the rate of PeT. The two VF dyes with intermediate baseline $\tau_{\mathrm{fl}}\left({ }^{\mathrm{i}} \mathrm{PrVF}[19]\right.$ and VF2.1.Cl) have the highest absolute sensitivities to $V_{\text {mem }}$ changes, while indicators in this series with high (NN26VF [20], VF2.0.Cl) and low (IndoVF [18], JuloVF [17]) baseline $\tau_{\mathrm{fl}}$ have much lower absolute sensitivities (Table 1). As previously noted, dim indicators can appear to have high relative sensitivity $(\% \Delta F / F)$, and we observe this trend for dyes with low baseline $\tau_{\mathrm{fl}}($ IndoVF [18], JuloVF [17], Table 1).

The performance of VFs hinges on a balance between the rates of PeT quenching and fluorescence. ${ }^{51}$ By tuning the extent of aniline conjugation with the molecular wire, which directly alters the HOMO energy levels, we present a strategy to optimize this balance. The $\tau_{\mathrm{fl}}$ of a fluorophore is the inverse of the 
sum of all of the rate constants for processes out of the excited state, including fluorescence $\left(k_{\mathrm{fl}}\right)$ and non-radiative pathways $\left(k_{\text {n.r. }}\right)$, which includes PeT $\left(k_{\mathrm{PeT}}\right) \cdot{ }^{32,52}$ Changes in $\tau_{\mathrm{fl}}$ in response to changes in $V_{\text {mem }}$ reflect modulation of the rate of PeT in VF dyes (Scheme 1a). We reason VFs with intermediate baseline $\tau_{\mathrm{fl}}$ exist in an optimal regime where the surrounding electric field of the cell membrane can modulate the rate of PeT with the highest dynamic range.

In the present VF dye library, indicators with intermediate baseline $\tau_{\mathrm{fl}}$ display the highest absolute sensitivity in physiological $V_{\text {mem }}$ ranges. Where the baseline $\tau_{\mathrm{fl}}$ is very short (JuloVF [17] and IndoVF [18]), the rate of PeT quenching is much larger than the rate of fluorescence. In these indicators, PeT quenching prevails regardless of $V_{\text {mem }}$-dependent changes in the rate of PeT, leading to low absolute sensitivity and a dim probe. Conversely, where baseline $\tau_{\mathrm{fl}}$ is longer (NN26VF [20]), PeT quenching is too slow and fluorescence prevails regardless of $V_{\text {mem }}$, producing a bright probe with low absolute sensitivity. The use of FLIM to measure $\tau_{\mathrm{fl}}$ provides an opportunity to explore the balance of the competing rates of fluorescence and PeT in living cells; ${ }^{51}$ reliance on fluorescence intensity, relative sensitivity $(\Delta F / F)$, and SNR precludes such an analysis, since intensity measurements are confounded by other factors beyond the photophysics of the indicator. ${ }^{32}$

We next examined how this balance between the rates of PeT and fluorescence translates to probe performance. We find a strong correlation between the absolute voltage sensitivity $\left(\Delta \tau_{\mathrm{fl}}\right.$ per $\left.\mathrm{mV}\right)$ and the signal-to-noise ratio (SNR) of a $100 \mathrm{mV}$ step in HEK293T (Fig. 5c and Tables 1, 2). The strong correlation between absolute voltage sensitivity and high SNR also exists for AP detection in excitable cells (Fig. 5d). Therefore, in the indicator series studied here, we can conclude that the balance between PeT and fluorescence is generally driving indicator performance. Where probes with similar lifetime properties performed differently (e.g. ${ }^{\mathrm{i}} \mathrm{PrVF}[19]$ and VF2.1.Cl), we can infer that differences in probe localization to the plasma membrane are likely playing a role. Therefore, we can conclude that the membrane localization of ${ }^{\mathrm{i}} \operatorname{PrVF}[19]$ would be a productive area to focus optimization of future derivatives.

We note that the intensity-based metric $\% \Delta F / F$ - or even HOMO energy level calculations - does not afford the same predictive or explanatory power. VF2.1.Cl has the highest SNR in both HEK293T and excitable cell systems, despite having a lower $\% \Delta F / F$ than three other VF dyes (Tables 1 and 2). HOMO energy levels, as a proxy for $\Delta G_{\mathrm{PeT}},{ }^{16}$ do not provide information about the balance between PeT and fluorescence. In other words, while $\% \Delta F / F$ (Fig. S3b, ESI $\dagger$ ) or baseline $\tau_{\mathrm{fl}}$ (Fig. S3c, ESI $\dagger$ ) correlate reasonably well with HOMO energy levels $\left(R^{2}=0.83\right.$ to 0.88$)$, none of these metrics correlate well with cellular performance (SNR) (Fig. S3d, ESI $\dagger$ ).

Overall, we find that absolute sensitivity of $\tau_{\mathrm{fl}}$ to $V_{\mathrm{mem}}$ is an excellent predictor of probe SNR in diverse contexts, capturing a snapshot of the balance between the rates of PeT and fluorescence. In addition, we demonstrate that absolute sensitivity $\left(\Delta \tau_{\mathrm{fl}}\right.$ per $\left.\mathrm{mV}\right)$ provides critical information for interpreting how structural modifications affect probe function and predicting probe performance in AP detection. Although SNR for a $100 \mathrm{mV}$ step in HEK293T cells also predicts SNR in AP detection, it offers far less information about the reason for the observed performance. For example, a low SNR may arise from a number of factors, from poor localization to the membrane, low quantum yield in lipid bilayers, or low voltage sensitivity. The use of $\tau_{\mathrm{fl}}$ in conjunction with intensity-based metrics yields a more complete understanding of existing VF properties and facilitates rational design of new indicators from these data.

\section{Guiding probe design with fluorescence lifetime}

In this work, we varied aniline conjugation to optimize action potential detection with VF dyes. We synthesized four new VoltageFluor indicators and discovered three new VoltageFluor dyes with higher $\Delta F / F$ values than the parent VF2.1.Cl. The new indicators JuloVF (17), IndoVF (18), and ${ }^{\mathrm{i}} \operatorname{PrVF}$ (19) have relative voltage sensitivities of 34,37 , and $34 \% \Delta F / F$ per $100 \mathrm{mV}$ in HEK293T cells (Fig. 2 and Table 1). We found that despite the higher nominal $\Delta F / F$ values of these new dyes, VF2.1.Cl displayed the best performance (SNR) in all applications we tested (Fig. 2-4 and Table 2).

Although the dimethyl aniline wire in VF2.1.Cl was the best molecular wire tested in this series with dichlorofluorescein, the same may not be true for red-shifted VFs. We previously showed that different wire scaffolds and HOMO energies are necessary to obtain good voltage sensitivity in VFs with redshifted chromophores. ${ }^{25-27}$ The aniline modifications that we present and characterize here offer an additional method for tuning the rate of PeT in VFs, enabling further optimization of red-shifted VF scaffolds.

One downside to $\Delta \tau_{\mathrm{fl}}$ per $\mathrm{mV}$ is that it requires considerable specialized equipment and effort to measure. As an alternative where many dyes are to be compared, the $\tau_{\mathrm{fl}}$ in POPC vesicles (Fig. 1, Table 1 and Fig. S17c, d, ESI $\dagger$ ) also appears to be a reasonable in vitro characterization strategy. We found that $\tau_{\mathrm{fl}}$ in POPC vesicles matched the trends observed in baseline lifetime in HEK293T cell membranes qualitatively, so intermediate values of $\tau_{\mathrm{fl}}$ in POPC vesicles may be a reasonable and rapid metric to examine in order to identify dyes that merit deeper analysis of $V_{\text {mem }}$ sensitivity.

\section{Summary}

In summary, we present the design and synthesis of a library of PeT-based voltage-sensitive dyes containing aniline groups with varying degrees of conjugation to the aromatic $\pi$ system. We performed extensive characterization of VF dye properties in vitro, in a model cell culture system, and in two different excitable cell types. The large range of brightness and voltage sensitivity observed in our library suggests that synthetic modification of the aniline electron donor is an effective way to tune PeT-based $V_{\text {mem }}$ sensing domains across a wide variety of electron donor strengths. We identify that $\tau_{\mathrm{fl}}$, and the magnitude of $V_{\text {mem }}$ induced changes in $\tau_{\mathrm{fl}}$, give a more accurate picture of $\mathrm{VF}$ photophysics than fluorescence intensity (SNR or $\Delta F / F$ ) alone. 
We anticipate that similar $\tau_{\mathrm{fl}}$ information would be useful for many novel probe libraries, not limited to those designed for $V_{\text {mem }}$ sensing.

\section{Conflicts of interest}

E. W. M. is listed as an inventor on a patent, owned by the Regents of the University of California, describing voltagesensitive fluorescent indicators.

\section{Acknowledgements}

We acknowledge the NIH for support (R35GM119855). S. C. B. and B. K. R. were supported in part by a grant from the NIH (T32GM066698). J. R. L.-D. was supported in part by an NSF Graduate Fellowship. $900 \mathrm{MHz}$ NMR were acquired at the Central California $900 \mathrm{MHz}$ NMR Facility, supported by NIH grant GM68933. We thank the Francis lab for use of their dynamic light scattering (DLS) instrument. We thank the College of Chemistry's NMR facility for resources provided and their staff for assistance. Instruments in CoC-NMR are supported in part by NIH S10OD024998. We thank Holly Aaron and Feather Ives for expert technical assistance with lifetime microscopy. FLIM experiments were performed at the UC Berkeley CRL Molecular Imaging Center, with instrumentation supported by NSF DBI-0116016.

\section{References}

1 C. M. Armstrong and W. F. Gilly, Access resistance and space clamp problems associated with whole-cell patch clamping, Methods Enzymol., 1992, 207, 100-122.

2 T. Knöpfel and C. Song, Optical voltage imaging in neurons: moving from technology development to practical tool, Nat. Rev. Neurosci., 2019, 20(12), 719-727.

3 E. W. Miller, Small molecule fluorescent voltage indicators for studying membrane potential, Curr. Opin. Chem. Biol., 2016, 33, 74-80.

4 M. Z. Lin and M. J. Schnitzer, Genetically encoded indicators of neuronal activity, Nat. Neurosci., 2016, 19(9), 1142-1153.

5 R. U. Kulkarni and E. W. Miller, Voltage Imaging: Pitfalls and Potential, Biochemistry, 2017, 56(39), 5171-5177.

6 Y. Xu, P. Zou and A. E. Cohen, Voltage imaging with genetically encoded indicators, Curr. Opin. Chem. Biol., 2017, 39, 1-10.

7 R. Y. Tsien, New calcium indicators and buffers with high selectivity against magnesium and protons: design, synthesis, and properties of prototype structures, Biochemistry, 1980, 19(11), 2396-2404.

8 G. Grynkiewicz, M. Poenie and R. Y. Tsien, A new generation of $\mathrm{Ca}^{2+}$ indicators with greatly improved fluorescence properties, J. Biol. Chem., 1985, 260(6), 3440-3450.

9 M. Fu, Y. Xiao, X. Qian, D. Zhao and Y. Xu, A design concept of long-wavelength fluorescent analogs of rhodamine dyes: replacement of oxygen with silicon atom, Chem. Commun., 2008, (15), 1780-1782.
10 Y. Koide, Y. Urano, K. Hanaoka, T. Terai and T. Nagano, Evolution of Group 14 Rhodamines as Platforms for NearInfrared Fluorescence Probes Utilizing Photoinduced Electron Transfer, ACS Chem. Biol., 2011, 6(6), 600-608.

11 X. Zhou, R. Lai, J. R. Beck, H. Li and C. I. Stains, Nebraska Red: a phosphinate-based near-infrared fluorophore scaffold for chemical biology applications, Chem. Commun., 2016, 52(83), 12290-12293.

12 X. Chai, X. Cui, B. Wang, F. Yang, Y. Cai, Q. Wu and T. Wang, Near-Infrared Phosphorus-Substituted Rhodamine with Emission Wavelength above $700 \mathrm{~nm}$ for Bioimaging, Chem. - Eur. J., 2015, 21(47), 16754-16758.

13 A. Fukazawa, S. Suda, M. Taki, E. Yamaguchi, M. Grzybowski, Y. Sato, T. Higashiyama and S. Yamaguchi, Phospha-fluorescein: a red-emissive fluorescein analogue with high photobleaching resistance, Chem. Commun., 2016, 52(6), 1120-1123.

14 J. Liu, Y.-Q. Sun, H. Zhang, H. Shi, Y. Shi and W. Guo, Sulfone-Rhodamines: A New Class of Near-Infrared Fluorescent Dyes for Bioimaging, ACS Appl. Mater. Interfaces, 2016, 8(35), 22953-22962.

15 J. B. Grimm, A. K. Muthusamy, Y. Liang, T. A. Brown, W. C. Lemon, R. Patel, R. Lu, J. J. Macklin, P. J. Keller, N. Ji and L. D. Lavis, A general method to fine-tune fluorophores for livecell and in vivo imaging, Nat. Methods, 2017, 14(10), 987-994.

16 Y. Urano, M. Kamiya, K. Kanda, T. Ueno, K. Hirose and T. Nagano, Evolution of Fluorescein as a Platform for Finely Tunable Fluorescence Probes, J. Am. Chem. Soc., 2005, 127(13), 4888-4894.

17 J. B. Grimm, B. P. English, J. Chen, J. P. Slaughter, Z. Zhang, A. Revyakin, R. Patel, J. J. Macklin, D. Normanno, R. H. Singer, T. Lionnet and L. D. Lavis, A general method to improve fluorophores for live-cell and single-molecule microscopy, Nat. Methods, 2015, 12(3), 244-250.

18 S.-n. Uno, M. Kamiya, T. Yoshihara, K. Sugawara, K. Okabe, M. C. Tarhan, H. Fujita, T. Funatsu, Y. Okada, S. Tobita and Y. Urano, A spontaneously blinking fluorophore based on intramolecular spirocyclization for live-cell super-resolution imaging, Nat. Chem., 2014, 6(8), 681-689.

19 H. Takakura, Y. Zhang, R. S. Erdmann, A. D. Thompson, Y. Lin, B. McNellis, F. Rivera-Molina, S.-N. Uno, M. Kamiya, Y. Urano, J. E. Rothman, J. Bewersdorf, A. Schepartz and D. Toomre, Long time-lapse nanoscopy with spontaneously blinking membrane probes, Nat. Biotechnol., 2017, 35(8), 773-780.

20 A. D. Thompson, M. H. Omar, F. Rivera-Molina, Z. Xi, A. J. Koleske, D. K. Toomre and A. Schepartz, Long-Term Live-Cell STED Nanoscopy of Primary and Cultured Cells with the Plasma Membrane HIDE Probe DiI-SiR, Angew. Chem., Int. Ed., 2017, 56(35), 10408-10412.

21 P. Liu and E. W. Miller, Electrophysiology, Unplugged: Imaging Membrane Potential with Fluorescent Indicators, Acc. Chem. Res., 2020, 53(1), 11-19.

22 E. Fluhler, V. G. Burnham and L. M. Loew, Spectra, membrane binding, and potentiometric responses of new charge shift probes, Biochemistry, 1985, 24(21), 5749-5755.

23 C. R. Woodford, E. P. Frady, R. S. Smith, B. Morey, G. Canzi, S. F. Palida, R. C. Araneda, W. B. Kristan, C. P. Kubiak, 
E. W. Miller and R. Y. Tsien, Improved PeT Molecules for Optically Sensing Voltage in Neurons, J. Am. Chem. Soc., 2015, 137(5), 1817-1824.

24 R. U. Kulkarni, D. J. Kramer, N. Pourmandi, K. Karbasi, H. S. Bateup and E. W. Miller, Voltage-sensitive rhodol with enhanced two-photon brightness, Proc. Natl. Acad. Sci. U. S. A., 2017, 114(11), 2813-2818.

25 Y.-L. Huang, A. S. Walker and E. W. Miller, A Photostable Silicon Rhodamine Platform for Optical Voltage Sensing, J. Am. Chem. Soc., 2015, 137(33), 10767-10776.

26 P. E. Deal, R. U. Kulkarni, S. H. Al-Abdullatif and E. W. Miller, Isomerically Pure Tetramethylrhodamine Voltage Reporters, J. Am. Chem. Soc., 2016, 138(29), 9085-9088.

27 G. Ortiz, P. Liu, S. H. H. Naing, V. R. Muller and E. W. Miller, Synthesis of Sulfonated Carbofluoresceins for Voltage Imaging, J. Am. Chem. Soc., 2019, 141(16), 6631-6638.

28 R. U. Kulkarni, H. Yin, N. Pourmandi, F. James, M. M. Adil, D. V. Schaffer, Y. Wang and E. W. Miller, A Rationally Designed, General Strategy for Membrane Orientation of Photoinduced Electron Transfer-Based Voltage-Sensitive Dyes, ACS Chem. Biol., 2017, 12(2), 407-413.

29 S. C. Boggess, S. S. Gandhi, B. A. Siemons, N. Huebsch, K. E. Healy and E. W. Miller, New Molecular Scaffolds for Fluorescent Voltage Indicators, ACS Chem. Biol., 2019, 14(3), 390-396.

30 E. W. Miller, J. Y. Lin, E. P. Frady, P. A. Steinbach, W. B. Kristan and R. Y. Tsien, Optically monitoring voltage in neurons by photo-induced electron transfer through molecular wires, Proc. Natl. Acad. Sci. U. S. A., 2012, 109(6), 2114-2119.

31 J. R. Lazzari-Dean, A. M. M. Gest and E. W. Miller, Optical estimation of absolute membrane potential using fluorescence lifetime imaging, eLife, 2019, 8, e44522.

32 M. Y. Berezin and S. Achilefu, Fluorescence Lifetime Measurements and Biological Imaging, Chem. Rev., 2010, 110(5), 2641-2684.

33 P. C. Lauterbur, C13 Nuclear Magnetic Resonance Spectroscopy. IV. Aniline, N,N-Dimethylaniline, and Their Methyl Derivatives: Steric Inhibition of Conjugation. The, J. Chem. Phys., 1963, 38(6), 1415-1431.

34 H. Ahlbrecht, E. O. Düber, J. Epsztajn and R. M. K. Marcinkowski, Delocalisation, conformation and basicity of anilines, Tetrahedron, 1984, 40(7), 1157-1165.

35 W. Kitching, I. D. Jonge, W. Adcock and A. N. Abeywickrema, Inhibition and enhancement of resonance as studied by $13 \mathrm{C}$ nuclear magnetic resonance spectroscopy, Org. Magn. Reson., 1980, 14(6), 502-510.

36 A. R. Katritzky, K. Yannakopoulou, P. Lue, D. Rasala and L. Urogdi, The chemistry of N-substituted benzotriazoles. Part 14. Novel routes to secondary and tertiary amines and to N, N-disubstituted hydroxylamines, J. Chem. Soc., Perkin Trans. 1, 1989, (2), 225-233.

37 D. Bourke and D. Collins, Synthesis and Some Reactions of 1-(Trimethoxymethyl)cyclohexene, Aust. J. Chem., 1996, 49(12), 1287-1291.

38 G. R. Fulmer, A. J. M. Miller, N. H. Sherden, H. E. Gottlieb, A. Nudelman, B. M. Stoltz, J. E. Bercaw and K. I. Goldberg,
NMR Chemical Shifts of Trace Impurities: Common Laboratory Solvents, Organics, and Gases in Deuterated Solvents Relevant to the Organometallic Chemist, Organometallics, 2010, 29(9), 2176-2179.

39 A. Kulkarni, W. Zhou and B. Török, Heterogeneous Catalytic Hydrogenation of Unprotected Indoles in Water: A Green Solution to a Long-Standing Challenge, Org. Lett., 2011, 13(19), 5124-5127.

40 D. Maiti, B. P. Fors, J. L. Henderson, Y. Nakamura and S. L. Buchwald, Palladium-catalyzed coupling of functionalized primary and secondary amines with aryl and heteroaryl halides: two ligands suffice in most cases, Chem. Sci., 2011, 2(1), 57-68.

41 S. E. Braslavsky, Glossary of terms used in photochemistry, 3rd edition (IUPAC Recommendations 2006), Pure Appl. Chem., 2007, 79(3), 293.

42 R. F. Chen and J. R. Knutson, Mechanism of fluorescence concentration quenching of carboxyfluorescein in liposomes: Energy transfer to nonfluorescent dimers, Anal. Biochem., 1988, 172(1), 61-77.

43 H. Ahlbrecht, E. O. Düber, J. Epsztajn and R. M. K. Marcinkowski, Delocalisation, conformation and basicity of anilines, Tetrahedron, 1984, 40, 1157-1165.

44 D. G. Bourke and D. J. Collins, Synthesis and some reactions of 1-(trimethoxymethyl)cyclohexene, Aust. J. Chem., 1996, 49, 1287-1291.

45 G. R. Fulmer, A. J. M. Miller, N. H. Sherden, H. E. Gottlieb, A. Nudelman, B. M. Stoltz, J. E. Bercaw and K. I. Goldberg, NMR chemical shifts of trace impurities: common laboratory solvents relevant to the organomettalic chemist, Organometallics, 2010, 29, 2176-2179.

46 A. R. Katritzky, K. Yannakopoulou, P. Lue, D. Rasala and L. Urogdi, The chemistry of $\mathrm{N}$-substituted benzotriazoles. Part 14. Novel routes to secondary and tertiary amines and to N,N-disubstituted hydroxylamines, J. Chem. Soc., Perkin Trans. 1, 1989, 225-233.

47 W. Kitching, I. D. Jonge, W. Adcock and A. N. Abeywickrema, Inhibition and enhancement of resonance as studied by $13 \mathrm{C}$ nuclear magnetic resonance spectroscopy, Org. Magn. Reson., 1980, 14(6), 502-510.

48 A. Kulkarni, W. Zhou and B. Török, Heterogeneous catalytic hydrogenation of unprotected indoles in water: A green solution to a long-standing challenge, Org. Lett., 2011, 13(19), 5124-5127.

49 A. Zakrzewska, R. Gawinecki, E. Kolehmainen and B. Ośmiałowski, 13C-NMR based evaluation of the electronic and steric interactions in aromatic amines, Int. J. Mol. Sci., 2005, 6(1), 52-62.

50 D. Rehm and A. Weller, Kinetics of Fluorescence Quenching by Electron and H-Atom Transfer, Isr. J. Chem., 1970, 8(2), 259-271.

51 L.-S. Li, Fluorescence Probes for Membrane Potentials Based on Mesoscopic Electron Transfer, Nano Lett., 2007, 7(10), 2981-2986.

52 J. R. Lakowicz, Principles of fluorescence spectroscopy, Kluwer Academic/Plenum, New York, 2nd edn, 1999. 